\title{
From Effective BCS Action to Vortex Dynamics.
}

\author{
G.E. Volovik \\ Low Temperature Laboratory \\ Helsinki University of Technology \\ Otakaari 3A, 02150 Espoo, Finland \\ and \\ L.D. Landau Institute for Theoretical Physics, \\ Kosygin Str. 2, 117940 Moscow, Russia
}

December 5, 2017

\begin{abstract}
The topological term in the effective action for the electrically neutral BCS system is discussed. It is applied for the calculation of the transverse force acting on the vortex in the limit of the smooth vortex core and vanishing interlevel distance in the vortex core. The controversy between the topological terms in cond-mat/9703124 and cond-mat/9411047 is resolved.
\end{abstract}

cond-mat/9703143 


\section{Introduction}

There are many papers devoted to the effective action for the BCS system. They differ by the topological terms: while in most of papers the topological term is $n \dot{\phi} / 2$, where $n$ is the particle density and $\phi$ is the phase of the order parameter (see eg [1] ), in the several other papers it is $\left(n-C_{0}\right) \dot{\phi} / 2$, where $C_{0}=p_{F}^{3} / 3 \pi^{2}$ is the particle density in the normal metal (see eg [2]). From these different topological actions different predictions are made for the transverse force, acting on the moving vortex. Here we discuss this controversy.

Actually both actions give the correct hydrodynamic equations for the BCS system in the uncharged limit. This is because the difference between the topological actions is the total time derivative if the parameter $C_{0}$ is treated as the dynamical invariant. This would mean that from both actions one should come to the same prediction for the vortex dynamics. However the latter depends on the proper treatment of the time derivative of the action. On the other hand, in the presence of the vortex, the phase $\phi$ is not defined globally, which makes means that the topological action in terms of $\phi$ is badly defined. That is why we propose another form of the topological term effective action, which does not contain the phase of the order parameter explicitly, and which follows from the gradient expansion of the effective action.

This action allows us to find the transverse force acting on the vortex in the special limit case, when the bosonic effective action provides the complete description of the vortex dynamics, ie in the case when the quantization of the levels in the vortex core can be neglected and the vortex core is smooth on the scale of the coherence length. In this limit our result for the transverse force coincides with that obtained in microscopic calculations [3].

\section{Effective BCS action at $T=0$.}

The calculated topological term in BCS action can be expressed in terms of the order parameter (gap function) $\Delta$ in the following form:

$$
S_{\text {top }}=\frac{1}{2 i} \int d^{3} r \int_{0}^{1} d \tau \int_{-\infty}^{\infty} d t \frac{\partial n}{\partial\left(|\Delta|^{2}\right)}\left(\frac{\partial \Delta}{\partial t} \frac{\partial \Delta^{*}}{\partial \tau}-\frac{\partial \Delta}{\partial \tau} \frac{\partial \Delta^{*}}{\partial t}\right)
$$


Here $\tau$ is an auxiliary coordinate, it is introduced when the effective action $\operatorname{Tr} \ln G^{-1}$ is presented as $\operatorname{Tr} \int_{0}^{1} d \tau G \partial_{\tau} G^{-1}$. The dependence on $\tau$ is chosen in such a way that at $\tau=0$ the coupling and the gap function are absent, $\left.\Delta(\vec{r}, t, \tau)\right|_{\tau=0}=0$, while $\tau=1$ corresponds to the physical $3+1$ dimensions, ie $\left.\Delta(\vec{r}, t, \tau)\right|_{\tau=1}=\Delta(\vec{r}, t)$. In Eq.(2.1) $n$ is the particle density, which depends on $\Delta$. At $\tau=0$ one has $n(\tau=0)=p_{F}^{3} / 3 \pi^{2} \equiv C_{0}$, which is the particle number density in the absence of the gap at the same chemical potential.

The quantity $n-C_{0}$ is small in the weak coupling limit of the BCS model, where it is determined by the particle-hole asymmetry, $n-C_{0} \sim n \Delta^{2} / E_{F}^{2} \ll$ $n$. In a smooth crossover from the BCS superconductivity to the condensate of the Cooper pairs, discussed in many papers (see recent papers [4, 5, 6), the parameter $C_{0}$ decreases and can become zero at some value of the coupling parameter $g: C_{0}(g)=0$ at $g>g_{c}$. In this case at $g=g_{c}$ one has the quantum (Lifshitz) transition at $T=0$. This zero temperature transition definitely happens if the quasiparticle spectrum has nodes, eg in the case of the $d$-wave Cooper pairing or in the $p$-wave state with the symmetry of the A-phase of superfluid ${ }^{3} \mathrm{He}$. In this case at $g=g_{c}$ the spectrum of quasiparticles is reconstructed - the nodes disappear (see Sections 6.2 and 9.4 in [7]). This is similar to the Lifshitz transition in metals, where the topology of Fermi surface changes. In what follows we assume that we are on the BCS (weak coupling) side, ie below the Lifshitz point, $g<g_{c}$.

In the BCS action the relevant variable is the the gap function $\Delta$, which means that all other variables, including $n$ are the functions of $|\Delta|^{2}$. The variation of the action over the order parameter is the surface integral in the 5 -dimensional space $(\mathbf{r}, \tau, t)$ and thus is expressed in terms of the physical coordinates $(\mathbf{r}, t)$ :

$$
\delta S_{\text {top }}=\frac{1}{2 i} \int d^{3} r \int_{-\infty}^{\infty} d t \frac{\partial n}{\partial\left(|\Delta|^{2}\right)}\left(\frac{\partial \Delta}{\partial t} \delta \Delta^{*}-\delta \Delta \frac{\partial \Delta^{*}}{\partial t}\right) .
$$

In principle from the variation of this topological action one can restore the action in the physical space-time, but the well defined action contains explicitely the parameter $C_{0}=n\left(|\Delta|^{2}=0\right)=p_{F}^{3} / 3 \pi$ :

$$
S_{\text {top }}=\frac{1}{4 i} \int d^{3} r \int_{-\infty}^{\infty} d t\left(n-n\left(|\Delta|^{2}=0\right)\right)\left(\frac{\dot{\Delta}^{*}}{\Delta^{*}}-\frac{\dot{\Delta}}{\Delta}\right) .
$$

If $\Delta(\mathbf{r}, t)$ is nowhere zero (this requirement excludes the case when vor- 
tices are present) one can introduce the phase $\phi$ of the order parameter:

$$
\Delta=|\Delta| e^{i \phi} .
$$

This allows to express the variation of the topological action in Eq.(2.2a) in terms of the canonically conjugated variables, $n$ and $\phi$ :

$$
\delta S_{\text {top }}=\frac{1}{2} \int d^{3} r \int_{-\infty}^{\infty} d t\left(\frac{\partial n}{\partial t} \delta \phi-\frac{\partial \phi}{\partial t} \delta n\right)
$$

where

$$
\delta n=\frac{\partial n}{\partial\left(|\Delta|^{2}\right)} \delta\left(|\Delta|^{2}\right)
$$

The action in Eq.(2.2b) can be also used in this case, it gives

$$
S_{\text {top }}=-\frac{1}{2} \int d^{3} r \int_{-\infty}^{\infty} d t\left(n-C_{0}\right) \frac{\partial \phi}{\partial t}
$$

This is what was obtained in [2].

The conventional contribution to the action is

$$
S_{0}=\int d^{3} r \int_{-\infty}^{\infty} d t\left(\frac{1}{2} m n_{s} \mathbf{v}_{s}^{2}+\epsilon\left(|\Delta|^{2}\right)\right),
$$

where $n_{s}$ is the superfluid density, $m$ is the mass of the fermion, $\mathbf{v}_{s}=\frac{\hbar}{2 m} \nabla \phi$ is the superfluid velocity and $\epsilon$ is the energy density, which depends on $|\Delta|^{2}$. Varying $S=S_{0}+S_{\text {top }}$ over $\phi$ and $|\Delta|^{2}$ one obtains the conventional hydrodynamic equations (we neglected the nonlinear terms in this procedure)

$$
\begin{gathered}
\frac{\partial n}{\partial t}+\nabla \cdot\left(n_{s} \mathbf{v}_{s}\right)=0 \\
\frac{\partial \phi}{\partial t}=-2 \mu \quad, \quad \mu=\frac{\partial \epsilon / \partial\left(|\Delta|^{2}\right)}{\partial n / \partial\left(|\Delta|^{2}\right)} .
\end{gathered}
$$

It is important here, that the hydrodynamic equations are general and do not contain the parameter $C_{0}$. This is because the term containing the factor $C_{0}$ in Eq. (2.4b) is the full derivative. In this sense there is no difference between the topological terms discussed in [1] and [2]. The difference becomes important when the zeroes in $\Delta$ appear and the phase of the order parameter is not defined globally any more. This is just the case of vortices. To find the vortex dynamics we must return to the action in Eq.(2.2a) for the order parameter, which does not contain the phase $\phi$ explicitly. 


\section{Effective action for the vortex dynamics in continuous limit.}

The effective action $S=S_{0}+S_{\text {top }}$ can be applied for the derivation of the dynamics of the vortex line only under certain conditions. We assume that the description in terms of the order parameter is complete, ie there is no other degrees of freedom. All the fermionic degrees of freedom are assumed to be inegrated off when the effective action was obtained. In this integration the fermionic spectrum was considered classically, ie the spectrum was taken as a function of the commuting spatial coordinate $\mathbf{r}$ and momentum $\mathbf{p}$ : $E=\sqrt{\varepsilon^{2}(\mathbf{p})+|\Delta(\mathbf{r})|^{2}}$. This means that if one applies this effective action to vortices one neglects the quantization of the fermions in the vortex background. This is justified only when the distance between the energy levels are small compared to the width of the levels, $\omega_{0} \tau \ll 1$. This represents the necessary condition for the application of the effective action to the vortex motion. Another important condition is that the core size of the vortex is to be large compared to the coherence length in order to neglect the higher order gradient terms in the action.

Under these conditions we can show that the BCS effective action leads to the following motion equation for the vortex:

$$
\pi N \hbar\left(n-C_{0}\right) \hat{z} \times \mathbf{v}_{L}=\pi N \hbar n_{s} \hat{z} \times \mathbf{v}_{s 0} .
$$

Here $\mathbf{v}_{L}$ is the vortex velocity with respect to the heat bath (normal component or crystal lattice), which is here assumed to be at rest; $\mathbf{v}_{s 0}$ is the superfluid velocity of the external superflow; $N$ is the winding number of the vortex. This coincides with the result of the microscopic calculations for the electrically neutral case in the limit of the large core size and in the regime $\omega_{0} \tau \ll 1[3]$.

To get the Eq.(3.1) from the BCS action let us introduce the vortex coordinate $\mathbf{r}_{L}(t)$. For simplicity we consider the rectilinear vortex along the axis $z$, so the order parameter $\Delta$ depends only on $\mathbf{r}-\mathbf{r}_{L}(t)$ where both vectors are 2-dimensional:

$$
\Delta(\mathbf{r}, t)=\Delta\left(\mathbf{r}-\mathbf{r}_{L}(t)\right)
$$


The variation of the topological action in Eq.(2.2) becomes

$$
\delta S_{t o p}=\frac{1}{2 i} \int d^{3} r \frac{\partial n}{\partial\left(||^{2}\right)}\left(\frac{\partial \Delta}{\partial x} \frac{\partial \Delta^{*}}{\partial y}-\frac{\partial \Delta}{\partial y} \frac{\partial \Delta^{*}}{\partial x}\right) \int_{-\infty}^{\infty} d t\left(\frac{\partial x_{L}}{\partial t} \delta y_{L}-\delta x_{L} \frac{\partial y_{L}}{\partial t}\right)
$$

For the axisymmetric vortex with

$$
\Delta(r, \varphi)=a(r) e^{i N \varphi}
$$

where $r$ and $\varphi$ are cylindrical coordinates, and $N$ is the winding number, one obtains

$$
\begin{gathered}
-i \int d^{3} r \frac{\partial n}{\partial\left(|\Delta|^{2}\right)}\left(\frac{\partial \Delta}{\partial x} \frac{\partial \Delta^{*}}{\partial y}-\frac{\partial \Delta}{\partial y} \frac{\partial \Delta^{*}}{\partial x}\right)= \\
=2 \pi N L \int_{0}^{\infty} d r \frac{\partial n}{\partial r}=2 \pi N L(n(\infty)-n(0))=2 \pi N L\left(n-C_{0}\right),
\end{gathered}
$$

where $L$ is the length of the straight vortex line. We took into account that on the vortex axis $(r=0)$ the order parameter is zero, $a(0)=0$, and therefore $n(0)=C_{0}$.

The same result can be obtained for any type of the vortex with the winding number $N$, because the volume integral can be transformed to the surface integral far from the vortex core, which is determined only by $N$ and $n\left(|\Delta(\infty)|^{2}\right)-n\left(|\Delta|^{2}=0\right)=n-C_{0}$. As a result

$$
\delta S_{\text {top }}=\pi \hbar N L\left(n-C_{0}\right) \int_{-\infty}^{\infty} d t\left(\frac{\partial x_{L}}{\partial t} \delta y_{L}-\delta x_{L} \frac{\partial y_{L}}{\partial t}\right)
$$

The variation of this topological action over $\delta \mathbf{r}_{L}$ gives the force acting on the vortex, which is represented by the term on the lhs of Eq.(3.1).

Note that the expression for the variation of Eq.(2.2a):

$$
\frac{\delta S_{t o p}}{\delta \mathbf{r}_{L}}=\frac{1}{2 i} \int d^{3} r \frac{\partial n}{\partial\left(|\Delta|^{2}\right)}\left(\frac{\partial \Delta}{\partial t} \nabla \Delta^{*}-\nabla \Delta \frac{\partial \Delta^{*}}{\partial t}\right)
$$

transforms to the Eq.(10) in [3] if one neglects the coordinate dependence of $\partial n / \partial\left(|\Delta|^{2}\right)$. The latter is justified only in the weak coupling limit, $g \ll$ 1 , explored in [3], while our approach is not limited by the weak coupling assumption: we assume only that $g<g_{c}$. 
In 8] the Eq.(10) of [3] is generalized to the case of the anisotropic pairing with the momentum dependent gap function, $\Delta(\mathbf{p}, \mathbf{r})$, but still in the weak coupling limit. We can generalize this to the arbitrary coupling strength introducing the momentum dependent particle distribution function

$$
n(\mathbf{p}, \mathbf{r})=\frac{1}{2}\left(1-\frac{\varepsilon(\mathbf{p})}{\sqrt{\varepsilon^{2}(\mathbf{p})+|\Delta(\mathbf{p}, \mathbf{r})|^{2}}}\right) \quad, \quad n(\mathbf{r})=2 \int \frac{d^{3} p}{(2 \pi)^{3}} n(\mathbf{p}, \mathbf{r}) \text {. }
$$

Then

$$
\begin{gathered}
\delta S_{t o p}=-i \int \frac{d^{3} p d^{3} r}{(2 \pi)^{3}} \int_{-\infty}^{\infty} d t \times \\
\times \frac{\partial n(\mathbf{p}, \mathbf{r})}{\partial\left(|\Delta(\mathbf{p}, \mathbf{r})|^{2}\right)}\left(\frac{\partial \Delta(\mathbf{p}, \mathbf{r})}{\partial t} \delta \Delta^{*}(\mathbf{p}, \mathbf{r})-\delta \Delta(\mathbf{p}, \mathbf{r}) \frac{\partial \Delta^{*}(\mathbf{p}, \mathbf{r})}{\partial t}\right)
\end{gathered}
$$

Applying the same procedure as before one obtains the Eq.(3.6) for any (smooth) structure of the vortex core and for arbitrary anisotropic pairing state. I am indebted to N.B. Kopnin for this remark [9].

The rhs term in Eq.(3.1) is obtained from the kinetic energy term in $S_{0}$ in Eq.(2.6). In the presence of the external superflow $\mathbf{v}_{s 0}$ the relevant term is

$$
\mathbf{v}_{s 0} \cdot \int d^{3} r n_{s} \mathbf{v}_{s}=\mathbf{v}_{s 0} \cdot \mathbf{P}
$$

The linear momentum $\mathbf{P}$ related to the vortex coordinate is

$$
\mathbf{P}=\pi \hbar N n_{s} L \hat{z} \times \mathbf{r}_{L}
$$

The variation of $\mathbf{v}_{s 0} \cdot \mathbf{P}$ over $\mathbf{r}_{L}$ gives the second term in Eq.(3.1).

The equation (3.1) is not Galilean invariant. The Galilean invariance is restored by introducing the velocity of the normal component $\mathbf{v}_{n}$, which coincides with the velocity of crystal lattice in the case of superconductors, or with the heat bath of the normal excitations in the case of superfluids. This equation can be represented as the balance of three forces acting on the vortex [10]:

$$
\pi \hbar N \hat{z} \times\left[n\left(\mathbf{v}_{s}-\mathbf{v}_{L}\right)+C_{0}\left(\mathbf{v}_{L}-\mathbf{v}_{n}\right)+\left(n-n_{s}\right)\left(\mathbf{v}_{n}-\mathbf{v}_{s}\right)\right]=0
$$

These are correspondingly the Magnus, the spectral flow and the Iordanskii forces in the terminology of Ref. 10. 
In the form of Eq.(3.1) the force balance was also obtained in [11], again

in the limit $\omega_{0} \tau \ll 1$; the first discussion of the lhs term in Eq.(3.1) as originating from the topological action was probably in [12 (see the paragraph containing the Eq.(10) in [12]).

\section{Discussion.}

In conclusion, the effective topological actions discussed in [1] and [2] are equivalent to each other in the particular case when the phase of the condensate is defined globally, and thus in the absence of vortices. In this case the two topological actions differ by the total time derivative and thus lead to the same hydrodynamic equations for the conjugated variables, the particle density $n$ and the condensate phase $\phi$. However these actions cannot be applied when the zeroes in the order parameter are present. In the presence of zeroes the topological action in Eq.(2.2) (or in Eq.(3.9) for the more general case of anisotropic pairing) is to be used, which resolves between the discussed actions [1] and [2] in favour of that in [2].

The Eq.(2.2) describes also the vortex dynamics in a special limit case. This dynamics agrees with the general phenomenological approach using the Poisson brackets scheme [13]. In this approach the phenomenological parameter $C_{0}$ is introduced as the dynamical invariant of the system, which does not violate the general properties of the hydrodynamical Poisson brackets. The BCS effective action for the neutral BCS system in Eq.(2.2) gives the precise value of this phenomenological parameter, $C_{0}=p_{F}^{3} / 3 \pi^{2}$, in the regime when the action is applicable for the vortex dynamics. The conditions of the applicability are $\omega_{0} \tau \ll 1$ and $R_{\text {core }} \gg \xi_{0}$, where $R_{\text {core }}$ is the core radius. They correspond to the vortex with the smooth core.

In the opposite limit $\omega_{0} \tau=\infty$ the effective action for the vortex dynamics in the BCS system can also be derived from the first principles exploring the Berry phase (see the recent paper [14]). In this case the discrete spectrum of the electrons in the core is of main importance. The properties of the exact wave functions of the electron in the potential produced by the vortex lead to the zero value of the canonical momentum density at the origin, $S(0)=0$, in [14]. This gives the zero value of the parameter $C_{0}$ in Eq.(3.12) in complete agreement with the result of [10], obtained in the limit $\omega_{0} \tau=\infty$. The finite life time $\tau$ destroys the coherence of the wave functions and finally restores 
the maximal value of $C_{0}$ in the limit $\omega_{0} \tau \ll 1$.

I would like to thank Jacek Dziarmaga, Nikolai Kopnin and Anne van Otterlo for stimulating discussions.

\section{References}

[1] I.J.R. Aitchison, P. Ao, D.J. Thouless and X.-M. Zhu, Phys. Rev., B 51, 6531 (1995).

[2] A. van Otterlo, D.S. Golubev, A.D. Zaikin and G. Blatter, condmat/9703124.

[3] N.B.Kopnin, Phys. Rev., B 54, 9475 (1996).

[4] V.B. Geshkenbein, L.B. Ioffe and A.I. Larkin, Phys. Rev., B 55, 3173 (1997).

[5] S. Stintzing and W. Zwerger, cond-mat/9703129.

[6] M. Marini, F. Pistolesi and G.C. Strinati, cond-mat/9703160.

[7] G. E. Volovik, Exotic properties of superfluid ${ }^{3} H e$, World Scientific, Singapore - New Jersey - London - Hong Kong, 1992.

[8] N.B.Kopnin and A.V. Lopatin, to be published in Phys. Rev. B.

[9] N.B. Kopnin, private communications.

[10] N.B.Kopnin, G.E.Volovik and Ü.Parts, Europhys. Lett. 32, 651 (1995).

[11] A. van Otterlo, M.V. Feigelman, V.B. Geshkenbein and G. Blatter, Phys. Rev. Lett. 75, 3736 (1995).

[12] G.E. Volovik, Pis'ma ZhETF 44, 144 (1986) [JETP Letters, 44, 185 (1986)].

[13] G.E. Volovik, Pis'ma ZhETF 64, 794 (1996) ( cond-mat/9610157).

[14] J. Dziarmaga, Phys. Rev., B 53, 6572 (1996). 